\title{
Association of the MAOA promoter UVNTR polymorphism with suicide attempts in patients with major depressive disorder
}

\author{
For-Wey Lung ${ }^{1,2,3}$, Dong-Sheng Tzeng ${ }^{1,4}$, Mei-Feng Huang ${ }^{5}$ and Ming-Been Lee
}

\begin{abstract}
Background: The MAOA uVNTR polymorphism has been documented to affect the MAOA gene at the transcriptional level and is associated with aggressive impulsive behaviors, depression associated with suicide (depressed suicide), and major depressive disorder (MDD). We hypothesized that the uVNTR polymorphism confers vulnerability to MDD, suicide or both. The aim of this study was to explore the association between the MAOA UVNTR and depressed suicide, using multiple controls.

Methods: Four different groups were included: 432 community controls, 385 patients with MDD who had not attempted suicide, 96 community subjects without mental disorders who had attempted suicide, and 109 patients with MDD who had attempted suicide. The MAOA uVNTR polymorphism was genotyped by a PCR technique. The symptom profiles and personal characteristics in each group were also compared.

Results: The MAOA 4R allele was more frequent in males with MDD than in male community controls $\left(\chi^{2}=4.182, p=\right.$ 0.041). Logistic regression analysis showed that, among the depressed subjects, those younger in age, more neurotic or who smoked had an increased risk of suicide $(\beta=-0.04, p=0.002 ; \beta=0.15, p=0.017 ; \beta=0.79, p=0.031$, respectively). Moreover, among those who had attempted suicide, those younger in age, with more paternal overprotection, and more somatic symptoms were more likely to be in the MDD group than in the community group $\beta=-0.11, p<0.001$; $\beta=0.15, p=0.026 ; \beta=1.11, p<0.001$ ). Structural equation modeling (SEM) showed that nongenetic factors, such as age, paternal overprotection, and somatic symptoms, were associated with MDD, whereas depressed suicide were associated with severity of depression, personality traits, age, marital status, and inversely associated with anxiety symptoms. However, depression did not affect suicidal behavior in the community group.
\end{abstract}

Conclusion: The MAOA 4R allele is associated with enhanced vulnerability to suicide in depressed males, but not in community subjects. The MAOA 4R allele affects vulnerability to suicide through the mediating factor of depressive symptoms. Further large-scale studies are needed to verify the psychopathology of the relationships among MAOA uVNTR polymorphism, symptom profiles, and suicidal behavior.

Keywords: MAOA u-VNTR Polymorphisms, Suicide Attempts, Major Depressive Disorder

\section{Background}

According to the results of a population-based survey, $16.3 \%$ of young adults in the general population in the USA and $25.2 \%$ of individuals with a general medical condition develop suicidal ideation [1]. In Taiwan, symptoms of depression have been reported to be one

\footnotetext{
* Correspondence: mingbeen@ntu.edu.tw

${ }^{6}$ Department of Psychiatry, National Taiwan University College of Medicine, Taipei, Taiwan

Full list of author information is available at the end of the article
}

of the three major predictors related to the development of suicidal ideation in psychiatric patients [2]. It has also been reported that serious suicidal behavior might occur more readily in those patients with mental illness or psychiatric comorbidity during their lifetime. However, the relationship between psychiatric comorbidity and the risk of suicide varies with age and gender [3]. Individuals sometimes also commit suicide in the absence of psychiatric disorders $[1,2]$. Furthermore, Lung and Lee [2] found that different factors contribute to the risk of
C Biomed Central

() 2011 Lung et al; licensee BioMed Central Ltd. This is an Open Access article distributed under the terms of the Creative Commons Attribution License (http://creativecommons.org/licenses/by/2.0), which permits unrestricted use, distribution, and reproduction in any medium, provided the original work is properly cited. 
suicide in different groups. Depression, hostility, and feelings of inferiority are predictors of suicide in patients with psychiatric illness, whereas hostility, feelings of inferiority, and insomnia are predictors of suicide in subjects from the community [2]. This demonstrates that the factors that affect attempted suicide in psychiatric patients and in the general population might differ.

In addition to the above-mentioned proximal factors, personal characteristics, which include gender, socioeconomic status, age, education, and personality, are important factors that are associated with suicidal behavior. Some studies have found that neuroticism is associated with increased rates of suicide $[4,5]$, whereas extraversion has a negative association [5]. Moreover, few studies of suicide have included the proximal psychological autopsy and distal predisposing factors among the factors investigated $[6,7]$.

A family history of suicidal behavior and certain genetic factors are linked to the development of suicidal ideation $[8,9]$. Increasingly, studies have indicated that gene-environment interactions might play a fundamental role in the occurrence of suicide attempts [10]. Abnormalities of the serotonergic system have been found to be involved in the risk of suicide. For example, the $102 \mathrm{C}$ allele of the 5 -HT2A serotonin receptor is associated with suicidal ideation in depressive patients, and the L/L (long/long) genotype of the 5-HTTLPR polymorphism of the serotonin transporter gene is associated significantly with completed suicide. In contrast, the S/S (short/short) genotype is found more frequently in individuals who have attempted suicide but failed, and who have further suicidal behavior, than in those who have not $[11,12]$.

The gene for monoamine oxidase A (MAOA), which is located on chromosome Xp21-p11 [13], is involved in the serotonergic pathway, and contains a region within its promoter that is polymorphic with respect to the number of copies of a 30-base pair repeat. The alleles that have been identified for this upstream variable number of tandem repeats (uVNTR) polymorphism include alleles with $3,3.5$, 4, and 5 repeats (3R, 3.5R, 4R, and 5R) [14]. These different $u$ VNTR variants are associated with different transcriptional activities of the MAOA promoter [15], which in turn result in different levels of expression of the MAOA gene. The allele of the MAOA uVNTR polymorphism that results in high levels of expression (high activity-related allele) has been reported to be associated in males with suicide caused by depression [16]. In addition, postmortem results have shown an increased level of MAOA activity in the hypothalamus of the brains of suicide victims with depressive disorder [17]. By contrast, in a population of healthy subjects from the community, male carriers of the low activity alleles ( $3 R$ and $5 R$ ) showed lower scores on a composite measure of dispositional aggressiveness and impulsivity, and showed a greater responsiveness to serotonin in the central nervous system (CNS), than men with the high activity allele [18].

The MAOA uVNTR polymorphism has been identified as a genetic factor that can modulate the risk for depression, suicide, or both by influencing monoaminergic activity in a sexually dimorphic manner [16]. However, little is known about whether the MAOA uVNTR polymorphism confers vulnerability to major depressive disorder (MDD) or suicidal behavior for the Han Chinese population in Taiwan.

Gene-gene interactions are common in human diseases. For example, the apolipoprotein (Apo) $\varepsilon 2$ allele is a potential confounding covariate for the MAOA uVNTR polymorphism in MDD: the high activity MAOA uVNTR alleles are associated with MDD when the Apo $\varepsilon 2$ allele, which is a protective factor for MDD, is adjusted for [19]. Moreover, gene-environment interactions are also an important factor. For example, Lin and colleagues also identified a sex-specific interaction between the MAOA uVNTR polymorphism and MDD [19]. In addition, maltreated children who carry the high activity alleles of the MAOA UVNTR are less likely to develop antisocial problems and behaviors than maltreated children who carry the low activity alleles [20].

Therefore, in this study, we aimed to investigate the role of the different MAOA variants in relation to MDD and/or attempted suicide. We used structural equation modeling (SEM) to investigate the relationships among personality traits, and other demographic factors, in participants with MDD and those from the community who had or had not attempted suicide.

\section{Methods}

\section{Sample Collection}

We recruited four groups of participants: 1) controls from the community, 2) patients with MDD, 3) subjects without mental disorders who had attempted suicide recruited from the emergency room, and 4) patients with MDD who had attempted suicide. A total of 1022 participants were recruited: the community control group included 432 participants; the MDD group, 385 participants; community subjects who had attempted suicide, 96 participants; and the group of patients with MDD who had attempted suicide, 109 participants. The patients with MDD were recruited from a teaching hospital in southern Taiwan from April 2001 to March 2006. All the participants were interviewed by two senior psychiatrists and research assistants to ensure that they did or did not meet the psychiatric diagnosis of MDD, according to the Diagnostic and Statistical Manual of Mental Disorders [21] using the Mini-international Neuropsychiatric Interview (MINI) [22]. 


\section{Materials}

Personality and symptom profiles were collected. Personality was assessed using the Eysenck Personality Questionnaire (EPQ), which is a 25-item self-report inventory that measures the personality traits of extraversion and neuroticism. The questionnaire comprises 14 neuroticism items, which measure the emotional dysfunction of an individual, and 11 extraversion items, which measure the sociability of an individual. The Chinese-language version of the EPQ has demonstrated a high internal consistency of 0.90 [23].

Symptom profiles were assessed using the Chinese Health Questionnaire (CHQ). The CHQ is a 12-item screening instrument that is used to identify minor psychiatric disorders in individuals in the community or in nonpsychiatric departments. The CHQ includes assessment of anxiety and depression. Cheng et al. [24] have demonstrated an internal consistency of 0.79 for the Chinese-language version of the CHQ.

The study protocol was approved by the Institutional Review Board of Kaohsiung Armed Forces General Hospital in southern Taiwan. Informed consent was obtained from all participants before enrollment.

\section{DNA Extraction}

Genomic DNA was extracted from $3.5 \mathrm{ml}$ of peripheral blood from each participant using a QIAamp DNA Blood Mini Kit (QIAGEN, Valencia, USA), in accordance with the manufacturer's protocol. The extracted DNA was stored at $-70^{\circ} \mathrm{C}$ until further use.

\section{Genotyping}

Amplification by the polymerase chain reaction (PCR) was performed in a final volume of $25 \mu \mathrm{l}$, which contained 50-100 ng of DNA, $50 \mathrm{mM} \mathrm{KCl,} 1.5 \mathrm{mM} \mathrm{MgCl}_{2}$, $200 \mu \mathrm{M}$ each of dATP, dCTP, and dTTP, $50 \mu \mathrm{M}$ dGTP, $150 \mu \mathrm{M}$ 7-deaza-dGTP, 10 pmol each of two primers (MAOA-F: 5'-ACAGCCTCGCCGTGGAGAAG-3' and MAOA-R: 5'-GAACGGACGCTCCATTCGGA-3'), and $1 \mathrm{U}$ of Taq polymerase (Biolab USA Inc, San Francisco, USA). The PCR was performed on a Hybaid PXE Thermal Cycler (Thermo Fisher Scientific, Barrington, USA) with the following cycling conditions: 35 cycles of denaturation at $94^{\circ} \mathrm{C}$ for $30 \mathrm{~s}$, annealing at $55^{\circ} \mathrm{C}$ for $30 \mathrm{~s}$, and extension at $72^{\circ} \mathrm{C}$ for $30 \mathrm{~s}$. The PCR products were subjected to gel electrophoresis on a $2.5 \%$ agarose gel (Amresco, Ohio, USA) with $0.5 \times$ TBE running buffer. The gels were stained with ethidium bromide and visualized using an ImageMaster VDS gel documentation system (Pharmacia Biotech, Uppsala, Sweden). The MAOA promoter UVNTR polymorphism was interpreted on the basis of the results of our previous study [25], in which the sizes of the PCR products for the $2 R, 3 R, 4 R$, and $5 R$ allelic variants were $320 \mathrm{bp}, 350 \mathrm{bp}, 380 \mathrm{bp}$, and 410 bp, respectively. The $2 \mathrm{R}$ and $3 \mathrm{R}$ allelic variants were defined as short-form variants, and the remaining variants, with a repeat number greater than three, were defined as long-form variants.

\section{Statistical Analysis}

In the descriptive analysis, continuous variables were expressed as the mean \pm SD and categorical variables were shown as proportions. We used the algorithm developed by Guo and Thompson [26], which allows an exact test to be performed for traits that are encoded by multiple alleles, to test whether the allelic frequency of the MAOA uVNTR polymorphism in females was in Hardy-Weinberg equilibrium. The Pearson's chi-square test was applied to distinguish differences among the four groups studied. Moreover, the allelic variants were categorized further into two groups according to their transcriptional activity, as described by Sabol et al. [14]. Genotypes that were homozygous and/or hemizygous for the $3 \mathrm{R}$ allele formed the low activity group, whereas those homozygous and/or hemizygous for the $4 R$ allele were assigned to the high activity group. Pearson's chisquare test was used to determine whether intergroup differences in allelic frequency between the $3 R$ and $4 R$ variants were significant. Binary logistic regression analysis was carried out to clarify which factors among MAOA uVNTR polymorphism, age, personality traits, gender, smoking, parental attachment, and mental health condition were associated with either suicide attempts in patients with MDD or depressive symptoms in individuals who had attempted suicide. $\mathrm{P}$ values less than 0.05 were considered statistically significant. These statistical analyses were carried out using the SPSS 17.0 software for Windows. SEM was performed using the AMOS 7.0 software for Windows to illustrate the interrelationships between the variables studied, which included personality scores, depression, anxiety, suicidal behavior, MDD, and the presence of the MAOA 3R allele. The criteria used to indicate that the null hypothesis model corresponded to the true structure were $p$ values greater than 0.05 and adjusted goodness-of-fit index (AGFI) greater than 0.9 .

\section{Results}

We detected the following MAOA uVNTR allelic variants among the participants: $2 \mathrm{R}, 3 \mathrm{R}, 4 \mathrm{R}$, and $5 \mathrm{R}$. We did not identify any $3.5 \mathrm{R}$ alleles among the subjects. The allelic frequencies and genotype distribution for the MAOA uVNTR polymorphism are shown by gender and group in Table 1 . The frequency of the $2 R$ and $5 R$ alleles was extremely low in each group. Three females with MDD carried at least one $2 \mathrm{R}$ allele and three community control females harbored one 5R allele. Among the male participants, there were two carriers of the $2 \mathrm{R}$ 
Table 1 Allelic frequencies and genotype distribution of the MAOA uVNTR polymorphism in the four groups of participants, namely, community controls, subjects from the community who had attempted suicide, and patients with major depressive disorder who had or had not attempted suicide

\begin{tabular}{|c|c|c|c|c|c|c|c|c|c|c|}
\hline \multirow[b]{2}{*}{ Group } & \multirow[b]{2}{*}{ Age } & \multirow[b]{2}{*}{$\mathrm{N}(\%)$} & \multicolumn{8}{|c|}{ Genotype distribution, N (\%) } \\
\hline & & & R2/R2 & R2/R3 & R2/R4 & R3/R3 & R3/R4 & R3/R5 & R4/R4 & R4/R5 \\
\hline Comm-contl & $45.36 \pm 13.91$ & 420 & & & & & & & & \\
\hline Male & & 182(43.33\%) & & & & & & & & \\
\hline Female & & $238(56.67 \%)$ & & & & $88(36.97 \%)$ & $108(45.38 \%)$ & $1(0.42 \%)$ & $38(15.97 \%)$ & $3(1.26 \%)$ \\
\hline MDD & $45.17 \pm 15.25$ & 379 & & & & & & & & \\
\hline Male & & $146(38.52 \%)$ & & & & & & & & \\
\hline Female & & $233(61.48 \%)$ & $1(0.43 \%)$ & $1(0.43 \%)$ & $1(0.43 \%)$ & $81(34.76 \%)$ & $109(46.78 \%)$ & & $40(17.17 \%)$ & \\
\hline Comm-suic & $45.48 \pm 20.53$ & 74 & & & & & & & & \\
\hline Male & & $36(48.65 \%)$ & & & & & & & & \\
\hline Female & & $38(51.35 \%)$ & & & & $11(28.95 \%)$ & $22(57.89 \%)$ & & $5(15.16 \%)$ & \\
\hline MDD-suic & $39.73 \pm 14.71$ & 104 & & & & & & & & \\
\hline Male & & $46(44.23 \%)$ & & & & & & & & \\
\hline \multirow[t]{2}{*}{ Female } & & $58(55.77 \%)$ & & & & $24(41.38 \%)$ & $26(44.83 \%)$ & & $8(13.79 \%)$ & \\
\hline & Allelic frequenc & $\mathrm{N}(\%)$ & & & Comm-con & & MDD & & Comm-suic & \\
\hline Group & R2 & R3 & R4 & R5 & $\chi^{2}$ & $P$ & $\chi^{2}$ & $P$ & $\chi^{2}$ & $P$ \\
\hline Comm-contl & & $396(60.18 \%)$ & $257(39.06 \%)$ & $5(0.76 \%)$ & & & & & & \\
\hline Male & & $111(60.98 \%)$ & $70(38.46 \%)$ & $1(0.54 \%)$ & & & & & & \\
\hline Female & & $285(59.87 \%)$ & 187(39.29\%) & $4(0.84 \%)$ & & & & & & \\
\hline MDD & $6(0.98 \%)$ & $344(56.21 \%)$ & $262(42.81 \%)$ & & 1.950 & 0.163 & & & & \\
\hline Male & $2(1.37 \%)$ & $72(48.32 \%)$ & $72(48.32 \%)$ & & 4.182 & $0.041^{*}$ & & & & \\
\hline Female & $4(0.86 \%)$ & $272(58.37 \%)$ & $190(40.77 \%)$ & & 0.220 & 0.639 & & & & \\
\hline Comm-suic & 2(1.79\%) & $63(56.25 \%)$ & $47(41.96 \%)$ & & 0.446 & 0.504 & 0.010 & 0.921 & & \\
\hline Male & $2(5.55 \%)$ & $19(52.78 \%)$ & $15(41.67 \%)$ & & 0.355 & 0.551 & 0.381 & 0.537 & & \\
\hline Female & & 44(57.89\%) & $32(42.11 \%)$ & & 0.169 & 0.681 & 0.026 & 0.872 & & \\
\hline MDD-suic & $2(1.23 \%)$ & $96(59.26 \%)$ & 64(39.51\%) & & 0.022 & 0.881 & 0.542 & 0.462 & 0.200 & 0.655 \\
\hline Male & $2(4.34 \%)$ & $22(47.83 \%)$ & $22(47.83 \%)$ & & 1.879 & 0.170 & 0.000 & 1.000 & 0.266 & 0.606 \\
\hline Female & & $74(63.79 \%)$ & $42(36.21 \%)$ & & 0.456 & 0.500 & 0.934 & 0.334 & 0.674 & 0.412 \\
\hline
\end{tabular}

comm-contl: community controls; MDD: subjects with major depressive disorder; Comm-suic: community subjects who had attempted suicide; MDD-suic: subjects with major depressive disorder who had attempted suicide; $\mathrm{N}$ : the number of genotyped subjects; $\chi^{2}$ : the chi-squared value from Pearson's uncorrected method; asterisk indicates statistical significance. The p-value was obtained by comparing the frequencies of R3 and R4 in each pair of groups; the frequencies of R2 and R5 were ignored because they were low in our population. Overall, the female-to-male ratio was 1.385 (568/410).

allele in each group, with the exception of the community control group. Only one carrier of the $5 \mathrm{R}$ allele, in the community control group, was found among the male participants. The $2 \mathrm{R}$ and $5 \mathrm{R}$ alleles were not included in subsequent analyses owing to their low frequencies in our studied cohorts. Among the female community controls, the genotype distribution of the MAOA uVNTR polymorphism was in Hardy-Weinberg equilibrium ( $p=0.367$, data not shown). Among the female participants in each group, the most frequent genotype was $3 R / 4 R$, followed by $4 R / 4 R$ and $3 R / 3 R$. The $3 \mathrm{R}$ allele was frequent in the male community controls (60.98\%) and community subjects who had attempted suicide $(52.78 \%)$, but not in the other two groups. In contrast, the frequency of the $4 \mathrm{R}$ allele was significantly higher in males with MDD than in male community controls $\left(\chi^{2}=4.182, \mathrm{p}=0.041\right)$. Likewise, the $4 \mathrm{R}$ allele was more frequent in male subjects with MDD who had attempted suicide than in male community controls ( $\mathrm{p}$ $=0.170$ ). This finding suggested that the high activity variant (4R) of the MAOA uVNTR polymorphism was more prevalent in male patients with MDD than in those without MDD. This might indicate an association between the $4 \mathrm{R}$ allele and depression.

On the basis of some previous studies [27,28], we subdivided the allelic variants further into dichotomous groups. The high activity group comprised female homozygotes for the $4 \mathrm{R}$ allele and male hemizygotes for $4 \mathrm{R}$, whereas the low activity group contained carriers of the $3 \mathrm{R}$ allele. The genotype distributions of the subjects who were homozygous or hemizygous for $3 \mathrm{R}$ vs. those who were homozygous or hemizygous for $4 R$ in the four groups studied are shown in Table 2 . The high activity variant occurred more frequently among the male MDD and male MDD subjects who had attempted suicide (50\% in both groups) than among the males in the other 
Table 2 The genotype distributions of homozygotes or hemizygotes for the $3 R$ and $4 R$ alleles of the MAOA uVNTR in the four groups of participants

\begin{tabular}{|c|c|c|c|c|c|c|c|c|c|c|}
\hline \multirow[b]{2}{*}{ Group } & \multicolumn{2}{|c|}{ Genotype, N (\%) } & \multicolumn{2}{|c|}{ Comm-contl } & \multicolumn{2}{|c|}{ MDD } & \multicolumn{2}{|c|}{ Comm-suic } & \multirow[b]{2}{*}{ OR } & \multirow[b]{2}{*}{ 95\% C.I. } \\
\hline & $3 \mathrm{R}-\mathrm{H}$ & 4R-H & $\chi^{2}$ & $P$ & $\chi^{2}$ & $P$ & $\chi^{2}$ & $P$ & & \\
\hline Comm-contl & 199(64.82\%) & 108(35.18\%) & & & & & & & & \\
\hline Male & $111(61.32 \%)$ & 70(38.68\%) & & & & & & & & \\
\hline Female & $88(69.84 \%)$ & $38(30.16 \%)$ & & & & & & & & \\
\hline MDD & 153(57.74\%) & $112(42.26 \%)$ & 3.016 & 0.082 & & & & & & \\
\hline Male & $72(50.00 \%)$ & $72(50.00 \%)$ & 4.182 & $0.041^{*}$ & & & & & 1.586 & $1.019-2.467$ \\
\hline Female & $81(66.94 \%)$ & $40(33.06 \%)$ & 0.240 & 0.624 & & & & & & \\
\hline Comm-suic & $30(60.00 \%)$ & $20(40.00 \%)$ & 0.434 & 0.510 & & & & & & \\
\hline Male & 19(55.88\%) & 15(44.12\%) & 0.355 & 0.551 & 0.381 & 0.537 & & & & \\
\hline Female & $11(68.75 \%)$ & $5(31.25 \%)$ & 0.008 & 0.929 & 0.021 & 0.885 & & & & \\
\hline MDD-suic & $46(60.53 \%)$ & $30(39.47 \%)$ & 0.487 & 0.485 & & & & & & \\
\hline Male & $22(50.00 \%)$ & $22(50.00 \%)$ & 1.879 & 0.170 & 0.000 & 1.000 & 0.266 & 0.606 & & \\
\hline Female & $24(75.00 \%)$ & $8(25.00 \%)$ & 0.329 & 0.566 & 0.763 & 0.382 & 0.211 & 0.646 & & \\
\hline
\end{tabular}

3R-H: homozygous or hemizygous for the 3-repeat allele; 4R-H: homozygous or hemizygous for the 4-repeat allele; OR: relative odds ratio; $95 \%$ C.I.: $95 \%$ confidence interval; a: odds ratio of $4 \mathrm{R}-\mathrm{H}$ to $3 \mathrm{R}-\mathrm{H}$ in relation to depression in male subjects.

groups (38.68-44.12\%). In addition, a significant difference with respect to the frequency of the $4 \mathrm{R}$ allele was found between male patients with MDD and male community controls, but no significant differences were found for the other pairs of groups. Given that the frequency of hemizygosity for the $4 R$ allele was the same in male patients with MDD and in male MDD-suicide patients (50\% vs. $50 \%$ ), it was unlikely that hemizygosity for the $4 R$ allele was associated with attempted suicide in male patients with MDD. This finding was basically consistent with the data that showed that male subjects who were hemizygous for the $4 \mathrm{R}$ allele had a 1.586 times higher risk of developing depression than male subjects who were hemizygous for the $3 \mathrm{R}$ allele.

Logistic regression was used to investigate the group of patients with MDD to determine which factors increased the risk of suicide: subjects with MDD who had attempted suicide were compared with subjects with MDD who had not. The results showed that subjects with MDD who were younger in age, more neurotic or who smoked had an increased risk of suicide $(\beta=$ -0.04, $\mathrm{p}=0.002 ; \beta=0.15, \mathrm{p}=0.017 ; \beta=0.79, \mathrm{p}=$ 0.031 , respectively), as shown in Table 3 . Differences in parental attachment, personality characteristics, mental health condition, demographics, and allelic distribution were also analyzed between the two groups who had attempted suicide, those with MDD and those without. The results showed that, for the two groups who had attempted suicide, subjects who were younger in age, had received more paternal care, and displayed more somatic symptoms were more likely to be in the MDD group than subjects who did not display these characteristics $(\beta=-0.11, \mathrm{p}<0.001 ; \beta=0.15, \mathrm{p}=0.026 ; \beta=1.11$, $\mathrm{p}<0.001$, respectively), as shown in Table 4 .
SEM was used to investigate the pathway relationships and to compare the factors of parental attachment, personality characteristics, mental health condition, demographics, and allelic distribution of the MAOA uVNTR polymorphism between the two groups of patients with MDD, those who had made a suicide attempt and those who had not. The parsimonious model showed a good fit, with a p value of 0.890 (greater than 0.05 ) and an AGFI of 0.942 (greater than 0.9), as shown in Figure 1. The $4 R$ allele of the MAOA uVNTR ( 0 referred to $4 R$ and 1 referred to $3 R$ in the SEM) did not have a direct effect on attempted suicide in subjects with MDD, but it did have an indirect effect through the mediating factor of depressive symptoms (MAOA-depression: $\beta=-0.12$, $\mathrm{p}=0.031$; depression-suicide: $\beta=0.32, \mathrm{p}<0.001)$.

\section{Table 3 Logistic regression results for parental} attachment, personality characteristics, demographic variables, and allelic frequencies that increase the risk of suicide in patients with major depressive disorder (MDD)

\begin{tabular}{|c|c|c|c|c|c|c|c|}
\hline & \multirow[t]{2}{*}{ B } & \multirow[t]{2}{*}{ S.E. } & \multirow[t]{2}{*}{ df } & \multirow[t]{2}{*}{ P-value } & \multirow[t]{2}{*}{$\operatorname{Exp}(B)$} & \multicolumn{2}{|c|}{$95 \% \mathrm{Cl}$} \\
\hline & & & & & & lower & upper \\
\hline Maternal care & -0.05 & 0.03 & 1 & 0.123 & 1.05 & 1.00 & 1.15 \\
\hline Maternal protection & -0.07 & 0.04 & 1 & 0.134 & 1.07 & 1.00 & 1.19 \\
\hline Paternal care & -0.02 & 0.03 & 1 & 0.604 & 1.02 & 0.96 & 1.10 \\
\hline Paternal protection & -0.03 & 0.04 & 1 & 0.481 & 1.03 & 0.96 & 1.12 \\
\hline Extraversion & 0.08 & 0.05 & 1 & 0.134 & 0.93 & 0.84 & 1.02 \\
\hline Neuroticism & 0.15 & 0.06 & 1 & 0.017 & 0.86 & 0.75 & 0.97 \\
\hline Sex & 0.53 & 0.39 & 1 & 0.174 & 0.58 & 0.27 & 1.03 \\
\hline Age & -0.04 & 0.01 & 1 & 0.002 & 1.04 & 1.02 & 1.08 \\
\hline Smoke & 0.79 & 0.37 & 1 & 0.03 & 0.45 & 0.23 & 1.02 \\
\hline MAOA UVNTR & -0.06 & 0.37 & 1 & 0.881 & 1.06 & 0.60 & 2.79 \\
\hline
\end{tabular}

Dependent variable: Group- 1: MDD with suicide attempt, 0: MDD without suicide attempt; MAOA uVNTR: 1: 3R, 0: 4R 
Table 4 Logistic regression results for parental attachment, personality characteristics, demographic variables, and allelic frequencies that increase the risk of major depressive disorder (MDD) in participants who had attempted suicide

\begin{tabular}{|c|c|c|c|c|c|c|c|}
\hline & \multirow[t]{2}{*}{ B } & \multirow[t]{2}{*}{ S.E. } & \multirow[t]{2}{*}{ df } & \multirow[t]{2}{*}{ P-value } & \multirow[t]{2}{*}{$\operatorname{Exp}(B)$} & \multicolumn{2}{|c|}{$95 \% \mathrm{Cl}$} \\
\hline & & & & & & lower & upper \\
\hline Maternal care & -0.05 & 0.04 & 1 & 0.246 & 1.05 & 0.97 & 1.14 \\
\hline Maternal protection & -0.10 & 0.06 & 1 & 0.081 & 1.10 & 0.99 & 1.23 \\
\hline Paternal care & -0.06 & 0.04 & 1 & 0.128 & 1.07 & 0.98 & 1.15 \\
\hline Paternal protection & -0.05 & 0.44 & 1 & 0.290 & 1.05 & 0.96 & 1.14 \\
\hline Extraversion & -0.02 & 0.06 & 1 & 0.757 & 1.02 & 0.90 & 1.16 \\
\hline Neuroticism & 0.08 & 0.08 & 1 & 0.283 & 0.92 & 0.80 & 1.07 \\
\hline Somatic symptoms & 0.25 & 0.16 & 1 & 0.124 & 0.78 & 0.57 & 1.07 \\
\hline Sex & 0.60 & 0.45 & 1 & 0.189 & 0.55 & 0.23 & 1.34 \\
\hline Age & -0.06 & 0.02 & 1 & $<0.001$ & 1.06 & 1.03 & 1.09 \\
\hline Smoke & 0.71 & 0.44 & 1 & 0.11 & 0.49 & 0.21 & 1.17 \\
\hline
\end{tabular}

Dependent variable: Group- 1: MDD with suicide attempt, 0: community with suicide attempt

Other factors that affected suicide attempts in patients with MDD directly included the demographic factors of level of education, age, and marital status, the personality characteristics of extraversion and neuroticism, and anxiety symptoms. Those patients with MDD who had a lower level of education $(\beta=-0.23, \mathrm{p}<0.001)$ or younger age $(\beta=-0.31, \mathrm{p}<0.001)$, were single $(\beta=$ $-0.13, p=0.053)$, more extraverted or more neurotic $(\beta$ $=0.19, \mathrm{p}=0.008 ; \beta=0.22, \mathrm{p}=0.002)$, or had fewer anxiety symptoms $(\beta=-0.16, p=0.045)$ had higher risks of attempting suicide than their counterparts.

The parsimonious SEM model that compared the two groups of people who had attempted suicide, namely those with MDD and those without, resulted in a good fit with a p-value of 0.175 and an AGFI of 0.935 , as shown in Figure 2. The factors that affected directly whether the participants who had attempted suicide had MDD or not were age and somatic symptoms: patients who had made a suicide attempt and had MDD were younger and showed more somatic symptoms than those without MDD $(\beta=-0.41, \mathrm{p}<0.001 ; \beta=0.46, \mathrm{p}<$ 0.001 , respectively).

\section{Discussion}

The results of our study showed that the MAOA 4R allele was associated with increased vulnerability to depression among the male subjects in our studied cohorts. This association was not detected in the logistic regression analysis. However, in SEM, the $4 \mathrm{R}$ allele of MAOA uVNTR was found to have an indirect effect on suicide attempts in patients with MDD through the mediating factor of depressive symptoms. Other than

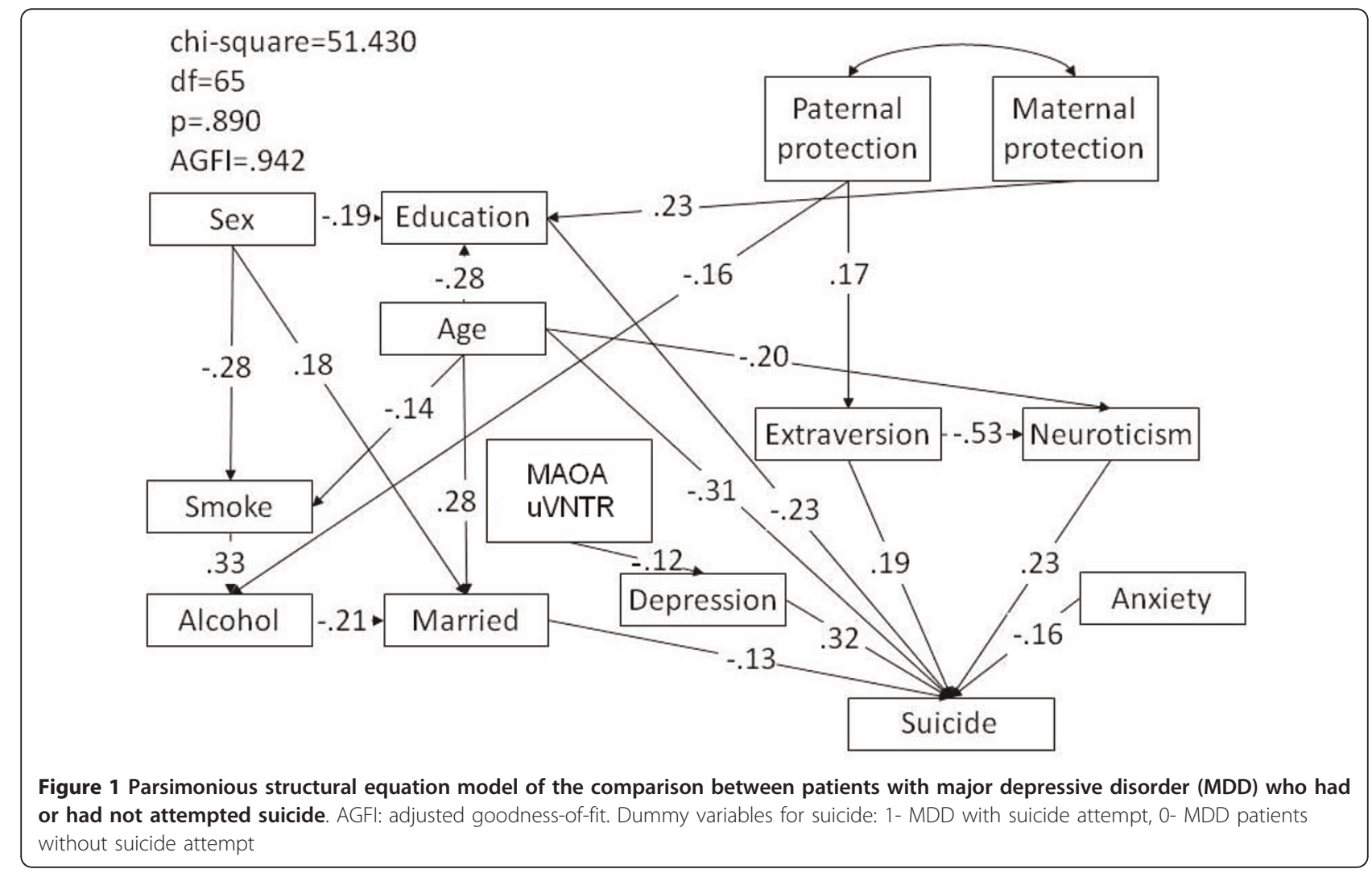




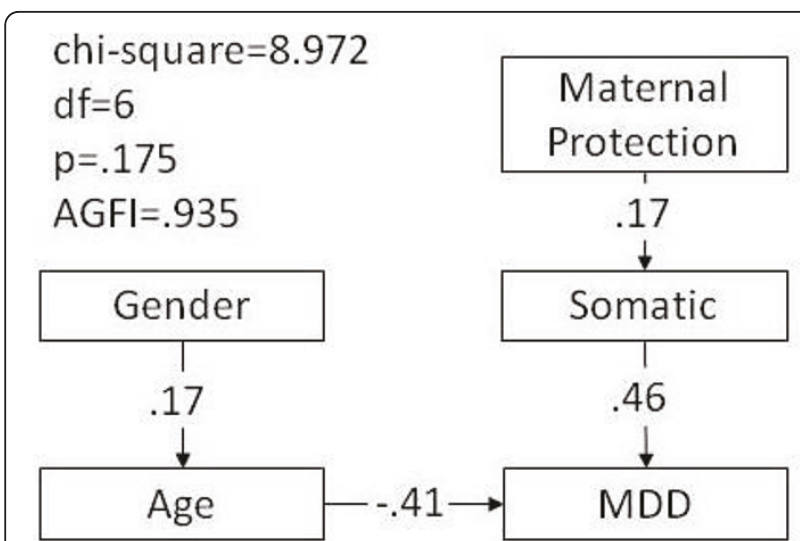

Figure 2 Parsimonious structural equation model of the comparison between participants who had attempted suicide with and without major depressive disorder (MDD). AGFI: adjusted goodness-of-fit. Dummy variables for suicide: 1- MDD with suicide attempt, 0 - community participant with suicide attempt

the length of the MAOA uVNTR polymorphism, additional factors that were associated with attempted suicide in MDD patients included the demographic factors of level of education, age, and marital status, the personality characteristics of extraversion and neuroticism, and anxiety symptoms. By contrast, among those who had attempted suicide, age and somatic symptoms were associated with depression.

In the present study, we detected four different alleles of the MAOA promoter uVNTR polymorphism $(2 \mathrm{R}, 3 \mathrm{R}$, $4 R$, and $5 R$ ), but we did not detect the $3.5 \mathrm{R}$ allele in our sample. It has been reported that the $3 R$ and $4 R$ alleles are the most common alleles among different ethnic populations [14]. In our population of 1022 participants, $71.29 \%$ of the participants in the community control group were found to carry at least one $3 \mathrm{R}$ allele, and $50.69 \%$ carried at least one $4 \mathrm{R}$ allele. By contrast, only nine participants carried the $2 \mathrm{R}$ allele and four carried the $5 \mathrm{R}$ allele. The allelic frequencies of the MAOA uVNTR polymorphism in our population were consistent with those described previously [14,19]. The $2 \mathrm{R}$ and $5 \mathrm{R}$ alleles were rare MAOA alleles in our populations; however, the frequency of the $2 \mathrm{R}$ allele seemed to be higher than that in Japanese and Caucasian people (0.2$1.3 \%$ vs. none), whereas the $5 \mathrm{R}$ allele was less common than in Caucasians $(0.2-0.76 \%$ vs. $1.6 \%)$. Interestingly, in this study, the $2 \mathrm{R}$ allele was detected in all groups except the community controls, whereas the $5 \mathrm{R}$ allele was observed only in the community control group. Experimental evidence from a cell-based assay, which contradicted the results from Sabol et al. and Deckert et al. [14,15], showed that the $2 R$ and $5 R$ alleles promote higher levels of luciferase reporter gene activity in two human cell lines (IMR-32 and Hela) than the other alleles [29]. Therefore, these two MAOA alleles might be involved in the biological functionality of the MAOA enzyme and possibly might be associated with disease, even though their frequencies are low.

The high activity $4 \mathrm{R}$ MAOA allele was found to be associated with depression in male subjects. This was partly consistent with the findings of a previous study by $\mathrm{Du}$ et al. [16]. In addition, males who were hemizygous for $4 \mathrm{R}$ were at higher risk $(\mathrm{OR}=1.586)$ of developing depressive symptoms than males who were hemizygous for 3R. We suspected that depressed males who carry the $4 \mathrm{R}$ allele might also be at higher risk of developing suicidal behavior than those who carry the 3R allele. Subjects who are homozygous/hemizygous for the MAOA $4 \mathrm{R}$ allele are assumed to have excess MAOA activity owing to increased transcription, which might result in abnormal serotonin metabolism in the CNS. Furthermore, the $4 \mathrm{R}$ allele of MAOA has been linked to high levels of aggressiveness and impulsivity, as well as poor responsiveness to serotonin in the CNS [18]. Thus, the higher risk of suicidal ideation or suicide attempts in subjects who harbor a high activity MAOA allele might be due to a high level of aggressiveness and impulsivity, and a low responsiveness to serotonin, in these individuals. Moreover, the MAOA uVNTR polymorphism has been recognized as one of four serotonin-related SNPs that might affect the decision-making ability of individuals, and thus modulate vulnerability to suicide [30].

From the intergroup comparisons, we found that hemizygosity for the $4 R$ allele was more common in depressed males, irrespective of whether they had attempted suicide. Thus, this result provides further evidence that the $4 \mathrm{R}$ allele can modulate the risk for depression, suicide or both by influencing monoaminergic signaling in a gender-specific manner. In addition, as mentioned above, it has been suggested that the highactivity MAOA allele is linked directly to high levels of aggression and impulsivity and low levels of responsiveness to serotonin in males without MDD. Therefore, we speculate that serotonergic signaling might be abnormal in male carriers of the $4 \mathrm{R}$ allele who are depressed. This abnormal signaling might result in a phenotype of aggression and impulsivity as a consequence of multifactorial interactions and play a role as risk factor for a suicide attempt. The logistic regression analysis that we carried out confirmed neither a significant association between the MAOA 4R allele and suicide nor an association between the MAOA 4R allele and depression. However, the results showed that subjects who were younger, more neurotic or who smoked had an increased risk of suicide. In addition, subjects who were younger, had received more paternal care, and had more somatic symptoms were more likely to be in the MDD group than their counterparts. This finding reveals the 
complexity of the involvement of the MAOA 4R allele in vulnerability to a suicide attempt or depression. As a consequence, SEM analysis was used to clarify the role of the $4 \mathrm{R}$ allele in the modulation of the risk of suicide and/or depression further, as well as to illustrate the interacting paths between the factors that were possibly associated.

Previous studies have shown inconsistencies with respect to the association between MAOA and suicide. Ono and colleagues [31] and Kunugi and colleagues [32] both failed to find an association between the MAOA polymorphism and suicide. However, Du et al. [16] showed that the high activity allele of MAOA was associated with depression-related suicide in male subjects, and indicated that the risk of a suicide attempt was 3.1 times greater among carriers of the high activity allele than among noncarriers. Our results were also consistent with the results from another study, which investigated Han Chinese subjects in Taiwan [33]. However, we only found borderline significance $(p=0.041)$ for the association between the $4 \mathrm{R}$ allele and MDD in male subjects on the basis of the direct observation of allelic frequencies. However, using SEM, we further determined that the MAOA $4 \mathrm{R}$ allele might be associated with suicide among patients with depression after controlling for other associated factors, such as gender, age, the personality characteristics of extraversion and neuroticism, and anxiety symptoms. The results indicated that the $4 \mathrm{R}$ allele has an indirect effect on suicide through the mediating effect of depressive symptoms. This effect was not detected when the subjects with MDD who had attempted suicide were compared with the subjects from the community who had attempted suicide. We rationalized that the indirect effect of the $4 R$ allele on suicide was due to the relatively high frequency of the $4 \mathrm{R}$ allele among the patients with MDD. Therefore, it might suggest that MAOA 4R allele was associated with enhanced risk of suicidal behavior among those MDD subjects. In contrast to our finding that the MAOA 4R allele was associated with suicide attempts in male patients with depression, the $4 \mathrm{R}$ allele was reported to be associated with MDD in German females [34]. The $4 \mathrm{R}$ allele was also reported to occur at a significantly higher frequency in female Chinese subjects with MDD than in controls [35]. In addition, the high activity allele was found at a significantly higher frequency in female subjects affected by mood disorders who presented a seasonal pattern or psychotic symptoms in their episodes than in individuals who did not show these characteristics [36]. Given that we found that the frequencies of the MAOA $4 \mathrm{R}$ and $3 \mathrm{R}$ alleles differed significantly between depressed males and male community controls, we conclude that carriage of the $4 \mathrm{R}$ allele by depressed male patients might make them more likely to attempt suicide than carriers of the $3 \mathrm{R}$ allele. Our results might explain why inaccurate diagnostic grouping, the lack of investigation of interaction effects, and uncontrolled potential confounding factors might yield inconsistent results when the intergroup differences in MAOA allelic frequencies are simply being observed by using a case-control experimental design.

Comparison of the two groups of patients with depression, those who had attempted suicide and those who had not, determined that, in addition to the MAOA 4R allele, depressive symptoms, lower level of education, younger age, and being more extraverted and more neurotic increased the risk of suicidal behavior. This result was consistent with the findings of a previous study, in which depressive symptoms were shown to be an important predictor for suicidal ideation in groups of patients with psychiatric or general medical conditions, but not in community controls [2]. Another study investigated subjects with MDD, and the severity of depression was identified as a predictor of suicidal ideation [37]. Several studies have shown that personality traits might be related to attempted suicide; for example, neuroticism shows a positive association $[4,5]$. The SEM model for subjects with and without MDD who had attempted suicide was also applied to the community participants (Figure 2); depression was not found to affect suicidal behavior in the community group. However, the SEM results showed that, among the subjects who had attempted suicide, younger subjects, together with those who had received more paternal care and displayed more somatic symptoms, were more likely to be in the MDD group. Taken together, these findings were consistent with our previous work, which showed that the risk factors for suicidal behavior differed between psychiatric patients and community subjects [2]. Thus, the hypothesis that depressive symptoms are associated with suicidal behavior in the general population is not supported by our study.

Furthermore, our study found an inverse relationship between anxiety and suicide. This is inconsistent with some previous studies which have found an association between anxiety and suicide [38]. However, other studies have also found otherwise $[39,40]$. Fawcett et al. [41] found that although anxiety was associated with suicide within the first year, but not with 2-10 years. Thus, different subtypes of MDD may be related to suicide. For instance, Grunebaum et al. [39] found the selfblame factor was related to suicide, but not anxiety measured using Hamilton Depression Rating Scales. One of the limitations of this study is that we used the $\mathrm{CHQ}$, which is only a general measure of psychiatric symptoms. Thus we were unable to identify the different subtypes of anxiety which may have different effect on suicide behavior. 
In the present study, we analyzed various factors that are associated with suicide, such as cigarette smoking and alcohol consumption, for intergroup differences. A statistically significant difference was found between the two groups of patients with MDD, those who had attempted suicide and those who had not, with respect to cigarette smoking: the participants with MDD who had attempted suicide smoked more cigarettes and drank more alcohol than those who had not attempted suicide. Similar findings have been reported for college students $[4,6]$ and patients with bipolar disorder [42]. According to previous studies, alcohol abuse, drug use, or smoking induces an increase in aggressive or impulsive behavior $[42,43]$. In a national comorbidity survey panel study, smoking was related to suicidal ideation or suicide attempts; however, it is likely that these associations are not related directly to the causal effects of smoking [44]. In fact, many genetic factors and behavioral traits have been shown to contribute to nicotine dependence in complex ways [45]. It has been documented that the MAOA 4R allele influences the smoking habits of females, as well as nicotine dependence and smoking initiation for male smokers in a Japanese population [46]. By contrast, in Chinese males, carriers of the 3R allele were reported to have a significantly increased risk of smoking as compared with carriers of the $4 R$ allele [47]. More recently, it has been demonstrated that smoking decreases the methylation of the MAOA promoter reliably when DNA extracted from lymphoblasts and whole blood samples is assayed [48]. This finding raises another issue: whether a possible interaction between smoking and the MAOA 4R allele affects not only nicotine dependence but also suicide in patients with depression through a decrease in MAOA methylation and the resultant gain-of-function effect. Further studies that are aimed at delineating the interrelationships among genotype-specific methylation, smoking history, current smoking status, gender, and MAOA promoter uVNTR are warranted.

The results of our study demonstrated that the MAOA 4R allele indirectly confers vulnerability to suicide in male patients with depression. We also produced a parsimonious model of the interrelationships among the factors that were associated with attempted suicide. However, our results should be viewed in the light of some experimental limitations. First, we did not investigate polymorphisms in other genes that are involved in the serotonergic pathway. The lack of this genetic information for data stratification might lead to bias, because the allelic variants of the MAOA uVNTR polymorphism are correlated with normal functionality of serotonin in the CNS. Second, the MAOA uVNTR variants are well known to be associated with different mental symptoms, and the genetic effect varies with gender. We performed data stratification using gender as a covariate. However, data stratification can result in false positive or false negative findings. Third, the community controls did not undergo a medical interview to assess their family history of psychiatric disorders; the required information was obtained simply from self-reports of the participants. Thus, the information gathered might possibly be incorrect. Fourth, survival effects might have influenced our results. However, individuals who had died as a result of suicide could not have been studied, and parasuicidal persons might have been omitted from the present study.

\section{Conclusion}

In conclusion, we have demonstrated that the $4 \mathrm{R}$ allele of the MAOA uVNTR polymorphism is associated with enhanced vulnerability to suicide in depressed males, but not in community subjects. Cross-sectional comparisons of nongenetic factors revealed that age, paternal care, and somatic symptoms are associated with MDD, whereas the severity of depression, personality traits (neuroticism, extraversion), age, marital status, and anxiety symptoms were associated with suicide in patients with depression. However, depression did not affect suicidal behavior in the community group. Further largescale studies are needed to verify the psychopathology of the relationships among the MAOA polymorphism, symptom profiles, including different subtypes of MDD, and attempts at suicide.

\section{Acknowledgements}

This study was supported by a grant (number DOH95-TD-M-113-046) from the National Health Research Institutes, Executive Yuan, Taiwan. We would like to express our appreciation to all the participants who agreed to provide biological samples and be interviewed. We also thank colleagues in our laboratory for their technical support, and the physicians and clinical nurses for their assistance and efforts.

Role of Funding Source

The funding organization had no role in the design or conduct of the study, in the collection, analysis, and interpretation of the data, or in the preparation, review, or approval of the manuscript.

\section{Author details}

${ }^{1}$ Department of Psychiatry, Kaohsiung Armed Forces General Hospital, Kaohsiung, Taiwan. ²Department of Neurology, Kaohsiung Medical University, Kaohsiung, Taiwan. ${ }^{3}$ Department of Psychiatry, National Defense Medical Center, Taipei, Taiwan. ${ }^{4}$ Graduate Institute of Undersea Medicine, National Defense Medical Center, Taipei, Taiwan. ${ }^{5}$ Department of Psychiatry, Kai-Suan Psychiatric Hospital, Kaohsiung, Taiwan. ${ }^{6}$ Department of Psychiatry, National Taiwan University College of Medicine, Taipei, Taiwan. ${ }^{7}$ Department of Social Medicine, National Taiwan University College of Medicine, Taipei, Taiwan. ${ }^{8}$ Director of Taiwan Suicide Prevention Center, Taipei, Taiwan.

\section{Authors' contributions}

All authors contributed to the design of the study. FW participated in the design of the study, performed the statistical analysis, and drafted the manuscript. DS participated in the design of the study, collected the data, and helped to draft the manuscript. MF carried out the molecular genetic studies and helped to draft the manuscript. MB conceived the study, participated in its design, and drafted the manuscript. All authors have reviewed and approved the manuscript. 


\section{Competing interests}

The authors declare that they have no competing interests.

Received: 28 July 2010 Accepted: 24 May 2011 Published: 24 May 2011

\section{References}

1. Druss B, Pincus H: Suicidal ideation and suicide attempts in general medical illnesses. Arch Intern Med 2000, 160(10):1522-1526.

2. Lung FW, Lee MB: The five-item Brief-Symptom Rating Scale as a suicide ideation screening instrument for psychiatric inpatients and community residents. BMC Psychiatry 2008, 8:53-53.

3. Beautrais AL, Joyce PR, Mulder RT, Fergusson DM, Deavoll BJ, Nightingale SK: Prevalence and comorbidity of mental disorders in persons making serious suicide attempts: a case-control study. Am J Psychiatry 1996, 153(8):1009-1014.

4. Gau SS-F, Lai MC, Chiu YN, Liu CT, Lee MB, Hwu HG: Individual and family correlates for cigarette smoking among Taiwanese college students. Compr Psychiatry 2009, 50(3):276-285.

5. Voracek M: Lack of association between digit ratio (2D:4D) and assertiveness: replication in a large sample. Percept Mot Skills 2009, 109(3):757-769.

6. Gau SS-F, Chen YY, Tsai FJ, Lee MB, Chiu YN, Soong WT, Hwu HG: Risk factors for suicide in Taiwanese college students. J Am Coll Health 2008, 57(2):135-142

7. McGirr A, Renaud J, Seguin M, Alda M, Benkelfat C, Lesage A, Turecki G: An examination of DSM-IV depressive symptoms and risk for suicide completion in major depressive disorder: a psychological autopsy study. J Affect Disord 2007, 97(1-3):203-209.

8. Brent DA, Mann JJ: Family genetic studies, suicide, and suicidal behavior. American journal of medical genetics 2005, 133C(1):13-24.

9. Du L, Bakish D, Lapierre YD, Ravindran AV, Hrdina PD: Association of polymorphism of serotonin $2 \mathrm{~A}$ receptor gene with suicidal ideation in major depressive disorder. Am J Med Genet 2000, 96(1):56-60.

10. Roy A, Sarchiopone M, Carli V: Gene-environment interaction and suicidal behavior. J Psychiatr Pract 2009, 15(4):282-288.

11. Courtet P, Picot MC, Bellivier F, Torres S, Jollant F, Michelon C, Castelnau D, Astruc B, Buresi C, Malafosse A: Serotonin transporter gene may be involved in short-term risk of subsequent suicide attempts. Biol Psychiatry 2004, 55(1):46-51.

12. Du L, Faludi G, Palkovits M, Bakish D, Hrdina PD: Serotonergic genes and suicidality. Crisis 2001, 22(2):54-60.

13. Ozelius L, Hsu YP, Bruns G, Powell JF, Chen S, Weyler W, Utterback M, Zucker D, Haines J, Trofatter JA, et al: Human monoamine oxidase gene (MAOA): chromosome position (Xp21-p11) and DNA polymorphism. Genomics 1988, 3(1):53-58.

14. Sabol SZ, Hu S, Hamer D: A functional polymorphism in the monoamine oxidase A gene promoter. Hum Genet 1998, 103(3):273-279.

15. Deckert J, Catalano M, Syagailo YV, Bosi M, Okladnova O, Di Bella D, Nothen MM, Maffei P, Franke P, Fritze J, et al: Excess of high activity monoamine oxidase A gene promoter alleles in female patients with panic disorder. Hum Mol Genet 1999, 8(4):621-624.

16. Du L, Faludi G, Palkovits M, Sotonyi P, Bakish D, Hrdina PD: High activityrelated allele of MAO-A gene associated with depressed suicide in males. Neuroreport 2002, 13(9):1195-1198.

17. Sherif F, Marcusson J, Oreland L: Brain gamma-aminobutyrate transaminase and monoamine oxidase activities in suicide victims. Eur Arch Psychiatry Clin Neurosci 1991, 241(3):139-144.

18. Manuck SB, Flory JD, Ferrell RE, Mann JJ, Muldoon MF: A regulatory polymorphism of the monoamine oxidase-A gene may be associated with variability in aggression, impulsivity, and central nervous system serotonergic responsivity. Psychiatry Res 2000, 95(1):9-23.

19. Lin CH, Chang YY, Lung FW: Sex-specific interaction between MAOA promoter polymorphism and Apo epsilon 2 allele in major depressive disorder in the Chinese population. Psychiatr Genet 2009, 19(6):337.

20. Caspi A, McClay J, Moffitt TE, Mill J, Martin J, Craig IW, Taylor A, Poulton R: Role of genotype in the cycle of violence in maltreated children. Science 2002, 297(5582):851-854

21. American-Psychiatric-Association: Diagnostic and statistical manual of mental disorders 4th edition. Washington, DC: American Psychiatric Association; 1994.
22. Sheehan DV, Lecrubier $Y$, Sheehan $K H$, Amorim $P$, Janavs J, Weiller $E$, Hergueta T, Baker R, Dunbar GC: The Mini-International Neuropsychiatric Interview (M.I.N.I.): the development and validation of a structured diagnostic psychiatric interview for DSM-IV and ICD-10. J Clin Psychiatry 1998, 59(Suppl 20):22-33, quiz 34-57.

23. Lu L: University transition: major and minor life stressors, personality characteristics and mental health. Psychol Med 1994, 24(1):81-87.

24. Cheng TA, WU JT, Chong MY, Williams P: Internal consistency and factor structure of the Chinese Health Questionnaire. Acta Psychiatr Scand 1990, 82(4):304-308.

25. Tzeng DS, Chien CC, Lung FW, Yang CY: MAOA gene polymorphisms and response to mirtazapine in major depression. Hum Psychopharmacol 2009, 24(4):293-300.

26. Guo SW, Thompson EA: Performing the exact test of Hardy-Weinberg proportion for multiple alleles. Biometrics 1992, 48(2):361-372.

27. Zhang H, Smith GN, Liu X, Holden JJ: Association of MAOA, 5-HTT, and NET promoter polymorphisms with gene expression and protein activity in human placentas. Physiological genomics 42(1):85-92.

28. Tsuchimine S, Yasui-Furukori N, Kaneda A, Saito M, Nakagami T, Sato K, Kaneko S: Association between monoamine oxidase A (MAOA) and personality traits in Japanese individuals. Progress in neuropsychopharmacology \& biological psychiatry 2008, 32(8):1932-1935.

29. Pai CY, Chou SL, Huag FFY: Assessment of the role of a functional VNTR polymorphism in MAOA gene promoter: a preliminary Study. Forensic Science Journal 2007, 6(2):37-43.

30. Jollant F, Buresi C, Guillaume S, Jaussent I, Bellivier F, Leboyer M, Castelnau D, Malafosse A, Courtet P: The influence of four serotoninrelated genes on decision-making in suicide attempters. Am J Med Genet B Neuropsychiatr Genet 2007, 144B(5):615-624.

31. Ono H, Shirakawa O, Nishiguchi N, Nishimura A, Nushida H, Ueno Y, Maeda K: No evidence of an association between a functional monoamine oxidase a gene polymorphism and completed suicides. Am J Med Genet 2002, 114(3):340-342.

32. Kunugi H, Ishida S, Kato T, Tatsumi M, Sakai T, Hattori M, Hirose T, Nanko S: A functional polymorphism in the promoter region of monoamine oxidase-A gene and mood disorders. Mol Psychiatry 1999, 4(4):393-395.

33. Huang SY, Lin MT, Lin WW, Huang CC, Shy MJ, Lu RB: Association of monoamine oxidase $A$ (MAOA) polymorphisms and clinical subgroups of major depressive disorders in the Han Chinese population. World J Biol Psychiatry 2009, 10 (4 Pt 2):544-551.

34. Schulze TG, Muller DJ, Krauss H, Scherk H, Ohlraun S, Syagailo YV, Windemuth C, Neidt H, Grassle M, Papassotiropoulos A, et al: Association between a functional polymorphism in the monoamine oxidase $A$ gene promoter and major depressive disorder. Am J Med Genet 2000, 96(6):801-803.

35. Yu YWY, Tsai SJ, Hong CJ, Chen TJ, Chen MC, Yang CW: Association study of a monoamine oxidase a gene promoter polymorphism with major depressive disorder and antidepressant response. Neuropsychopharmacology 2005, 30(9):1719-1723.

36. Gutierrez B, Arias B, Gasto C, Catalan R, Papiol S, Pintor L, Fananas L: Association analysis between a functional polymorphism in the monoamine oxidase A gene promoter and severe mood disorders. Psychiatr Genet 2004, 14(4):203-208.

37. Sokero TP, Melartin TK, Rytsala HJ, Leskela US, Lestela-Mielonen PS, Isometsa ET: Suicidal ideation and attempts among psychiatric patients with major depressive disorder. J Clin Psychiatry 2003, 64(9):1094-1100.

38. Bolton JM, Belik SL, Enns MW, Cox BJ, Sareen J: Exploring the correlates of suicide attempts among individuals with major depressive disorder: findings from the national epidemiologic survey on alcohol and related conditions. J Clin Psychiatry 2008, 69(7):1139-1149.

39. Grunebaum MF, Keilp J, Li S, Ellis SP, Burke AK, Oquendo MA, Mann JJ: Symptom components of standard depression scales and past suicidal behavior. J Affect Disord 2005, 87(1):73-82.

40. Grucza RA, Przybeck TR, Spitznagel EL, Cloninger CR: Personality and depressive symptoms: a multi-dimensional analysis. J Affect Disord 2003, 74(2):123-130

41. Fawcett J, Scheftner WA, Fogg L, Clark DC, Young MA, Hedeker D, Gibbons R: Time-related predictors of suicide in major affective disorder. Am J Psychiatry 1990, 147(9):1189-1194. 
42. Ostacher MJ, Lebeau RT, Perlis RH, Nierenberg AA, Lund HG, Moshier SJ, Sachs GS, Simon NM: Cigarette smoking is associated with suicidality in bipolar disorder. Bipolar Disord 2009, 11(7):766-771.

43. Breslau N, Schultz LR, Johnson EO, Peterson EL, Davis GC: Smoking and the risk of suicidal behavior: a prospective study of a community sample. Arch Gen Psychiatry 2005, 62(3):328-334.

44. Kessler RC, Borges G, Sampson N, Miller M, Nock MK: The association between smoking and subsequent suicide-related outcomes in the National Comorbidity Survey panel sample. Mol Psychiatry 2009, 14(12):1132-1142.

45. Hiroi N, Scott D: Constitutional mechanisms of vulnerability and resilience to nicotine dependence. Mol Psychiatry 2009, 14(7):653-667.

46. Ito H, Hamajima N, Matsuo K, Okuma K, Sato S, Ueda R, Tajima K. Monoamine oxidase polymorphisms and smoking behaviour in Japanese. Pharmacogenetics 2003, 13(2):73-79.

47. Jin Y, Chen D, Hu Y, Guo S, Sun H, Lu A, Zhang X, Li L: Association between monoamine oxidase gene polymorphisms and smoking behaviour in Chinese males. The international journal of neuropsychopharmacology/official scientific journal of the Collegium Internationale Neuropsychopharmacologicum (CINP) 2006, 9(5):557-564.

48. Philibert RA, Beach SR, Gunter TD, Brody GH, Madan A, Gerrard M: The effect of smoking on MAOA promoter methylation in DNA prepared from lymphoblasts and whole blood. Am J Med Genet B Neuropsychiatr Genet 153B(2):619-628

\section{Pre-publication history}

The pre-publication history for this paper can be accessed here: http://www.biomedcentral.com/1471-2350/12/74/prepub

doi:10.1186/1471-2350-12-74

Cite this article as: Lung et al: Association of the MAOA promoter uVNTR polymorphism with suicide attempts in patients with major depressive disorder. BMC Medical Genetics 2011 12:74.

\section{Submit your next manuscript to BioMed Central and take full advantage of:}

- Convenient online submission

- Thorough peer review

- No space constraints or color figure charges

- Immediate publication on acceptance

- Inclusion in PubMed, CAS, Scopus and Google Scholar

- Research which is freely available for redistribution

Submit your manuscript at www.biomedcentral.com/submit 